\title{
Photon harvesting with multi wall carbon nanotubes
}

\author{
M. Scarselli ${ }^{\text {a,* }}$, C. Scilletta ${ }^{\text {a }}$, F. Tombolini ${ }^{\text {a }}$, P. Castrucci ${ }^{a}$, M. De Crescenzi ${ }^{\text {a }}$, \\ M. Diociaiuti ${ }^{\text {b }}$, S. Casciardi ${ }^{\mathrm{c}}$, E. Gatto $^{\mathrm{d}}$, M. Venanzi $^{\mathrm{d}}$ \\ a Physics Department, University of Rome 'Tor Vergata', 00133 Roma, Italy \\ ${ }^{\mathrm{b}}$ Health and Technology Department, Istituto Superiore di Sanità, 00161 Roma, Italy \\ ${ }^{\mathrm{c}}$ Department of Occupational Hygiene, National Institute of Prevention and Safety at Workplace (ISPESL), 00040 Monte Porzio \\ Catone, Italy \\ d Chemistry Department, University of Rome 'Tor Vergata', 00133 Roma, Italy
}

\section{A R T I C L E I N F O}

Article history:

Available online 23 February 2009

\section{Keywords:}

Carbon nanotube

Hybrid material

Metal nanoparticle

Photocurrent

\section{Introduction}

Nanoscale carbon materials provide new ways to design energy conversion devices. In particular, the one-dimensional carbon nanotubes (CNTs) [1] superstructure offers the advantage of accessing the largest active surface for photon absorption and thus harvesting solar energy $[2,3]$. The presence of extended, delocalized $\pi$-electron systems is, indeed, very useful for charge transfer and transport. Investigations have been carried out regarding the photo-electrochemical properties of single wall carbon nanotubes (SWCNTs) and the photon-to-photocurrent efficiency (IPCE) [4] reported remained low (about $0.15 \%$ ) due to the rapid exciton annihilation [5]. For this reason a number of strategies were developed to enhance the photoconversion efficiency for SWCNTs. One method consisted in

\footnotetext{
* Corresponding address: Physics Department, CNISM, University of Rome 'Tor Vergata', 00133 Roma, Italy. Tel.: +39 0672594116 ; fax: +39 062023507.

E-mail address: manuela.scarselli@roma2.infn.it (M. Scarselli).
} 
simply covalently linking ordered molecular assemblies on the SWCNTs walls [6,7]. Results were also obtained from the dispersion of semiconducting quantum dots [8-12] and metallic nanoparticles [1316] on the SWCNTs and MWCNTs sidewalls. Photoelectrochemical measurements demonstrated that SWCNTs may act both as promoter for charge transfer and as charge transport from the excited molecular species. In this regard, a great deal of research has been devoted to trying to comprehend the modification of the electronic properties of such hybrid carbon systems as a function of the type, size and shape of the dispersed metal nanoparticles.

Because of their numerous concentric cylindrical walls, Multi-wall carbon nanotubes (MWCNTs) offer additional pathways for the photo excited charge to the collecting electrode. Nevertheless, the obvious challenge is to use a MWCNT network in a solar cell as a scaffold to anchor light-harvesting metal nano-particles and facilitate the electron transport to the collecting electrode surface.

Recently we have demonstrated that MWCNTs can generate photocurrent in the visible and ultraviolet spectral range [17]. In this work we show that a remarkable increase in the photocurrent signal can be obtained from the deposition of $\mathrm{Cu}$ nanoparticles on the sidewalls of MWCNTs. This effect was obtained from experiments made both with a photoelectrochemical cell and with a solid state device. The photocurrent signal enhancement was detected over the entire visible and near ultraviolet energy range, proving to be an efficient functionalization method as that obtained from SWCNT's modified with molecular assemblies [6,7].

\section{Experimental}

A silicon dioxide $\left(\mathrm{SiO}_{2}\right)$ substrate (Siltronix) of $100 \mathrm{~nm}$, thermally grown on $\mathrm{Si}(100)$ was used as a starting substrate for the carbon nanotubes growth. The substrate was inserted into the growth chamber operating in ultra high vacuum conditions (UHV) and an iron (Fe) catalyst was deposited, at room temperature, on the clean oxide substrate by thermal evaporation from a tungsten basket at a base pressure of $1 \times 10^{-8}$ Torr during the deposition. The nominal Fe thickness estimated with a quartz microbalance was about $1.00 \pm 0.01 \mathrm{~nm}$. Subsequently, carbon nanotubes were grown by thermal chemical vapor deposition (CVD) in acetylene $\left(\mathrm{C}_{2} \mathrm{H}_{2}\right)$ atmosphere (700 Torr pressure, for 30 min) while keeping the substrate covered with Fe nano-islands at $T=1073 \mathrm{~K}$ during the growth.

Soon after the growth of MWCNTs, we deposited Cu by thermal evaporation from a tungsten crucible in the same growth chamber at a base pressure of $1 \times 10^{-8}$ Torr. The nominal Cu thickness, estimated with a quartz microbalance was about $0.10 \pm 0.01 \mathrm{~nm}$.

Scanning Electron Microscopy (SEM) and Transmission Electron Microscopy (TEM) measurements were used to characterize the morphology, purity and the internal structure of the carbon nanotubes.

SEM images were recorded using a Cambridge 360 SteroScan apparatus (10 keV) directly on the $\mathrm{SiO}_{2}$ substrate where MWCNTs and $\mathrm{Cu}-\mathrm{MWCNT}$ were grown.

TEM measurements were performed in a FEI TECNAI 12 (120 keV) apparatus equipped with an energy filter (GATAN GIF model) and a Peltier cooled SSC (slow scan charged-coupled device) multiscan camera (794IR model). A droplet of the raw synthesis product diluted in isopropyl alcohol was dispersed on a gold TEM grid (mesh 1000, pitch 25 micron and thickness less than 10 micron). Most of the reaction products were thereby located next to or bridged between the gold wires.

Photo-electrochemical measurements on $\mathrm{MWCNTs} / \mathrm{SiO}_{2}$ and $\mathrm{Cu}-\mathrm{MWCNTs} / \mathrm{SiO}_{2}$ electrodes were carried out in a conventional three-electrodes electrochemical cell by using platinum (Pt) wire as counter electrode and a standard calomel electrode (SCE) as a reference electrode. A $0.5 \mathrm{M}$ NaI and $0.01 \mathrm{M} \mathrm{I}_{2}$ in acetonitrile solution was used as electrolyte. A $200 \mathrm{~W}$ Xe lamp (Osram) was employed as excitation source $(\lambda>300 \mathrm{~nm})$. The intensity of the light near the electrode surface was estimated by azobenzene actinometry [18]. Photocurrent measurements were carried out using the Xe lamp and a monochromator to select the wavelength of excitation and with a PG-310 (HEKA ELEKTRONIK) potentionstat. The immersed area of the sample irradiated was about $0.7 \mathrm{~cm}^{2}$ for both MWCNTs and Cu-MWCNTs samples and was estimated in situ before and after the photocurrent measurements. This value has been used in the evaluation of the IPCE.

The optical measurements were performed with a chopped radiation source and a lock-in technique. The source was an $150 \mathrm{~W}$ Xenon arc lamp and the radiation energy was selected by a f/3.4 monochromator ranging from $1.55 \mathrm{eV}$ up to $3.5 \mathrm{eV}$. The incident light was also modulated at $400 \mathrm{~Hz}$ 
a

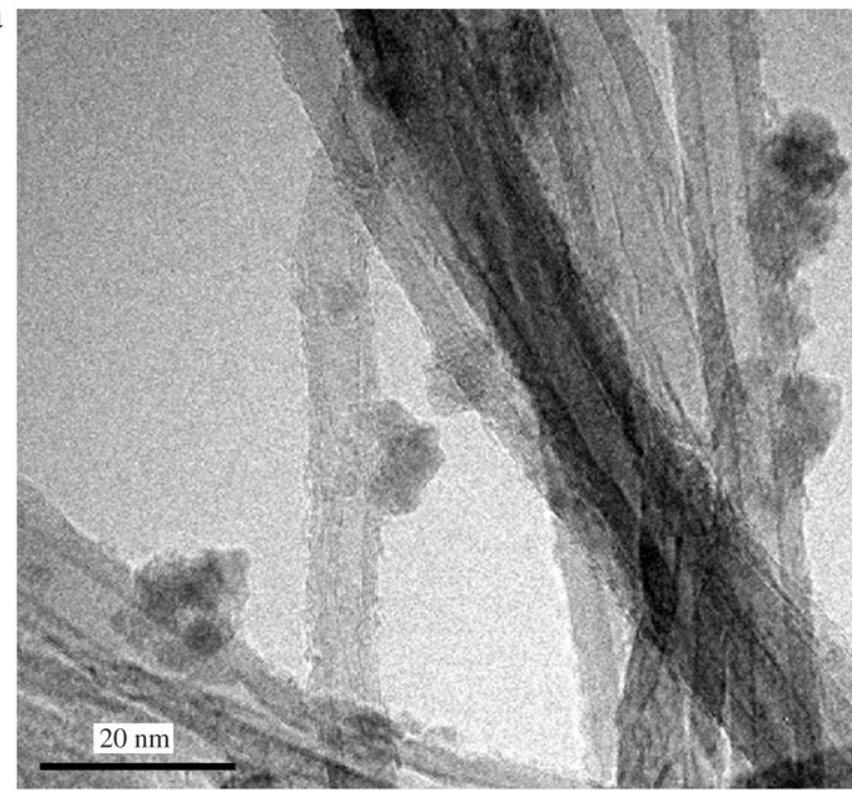

b

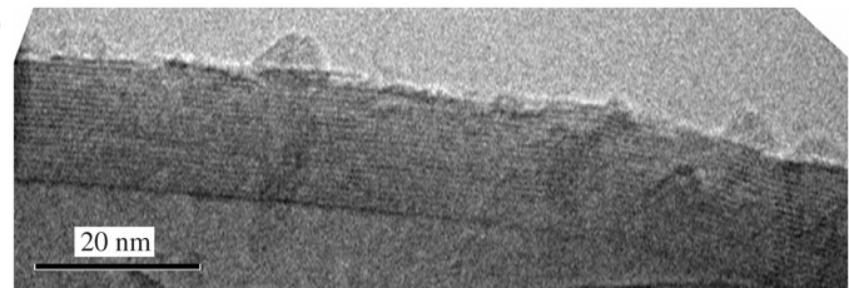

Fig. 1. TEM image obtained on MWCNT after Cu deposition.(a) large scale image obtained on a suspended group of Cu-MWCNT; (b) high resolution image obtained on a single Cu-MWCNT. The nanoparticles shape result mostly faceted with an average diameter of about $3.0 \pm 1.0 \mathrm{~nm}$.

through an optical chopper before reaching the sample surface. The chopper modulation frequency was also sent to a Signal Recovery DSP Lock-in Amplifier as reference for the measurement acquisition. The excitation light was monitored by a calibrated Silicon diode. The current signal coming from the sample was amplified by a Femto DLCPA-220 current preamplifier.

Two planar Ag metal contacts were made on the sample surface, only one of them was grounded. During the acquisition the spotlight was positioned close to the non grounded metal contact electrode and the current signal was recorded as a function the incident photon energy [19].

The set-up for measuring the modulated photocurrent is shown schematically in Fig. 3(a).

\section{Results and discussion}

The MWCNTs and Cu-MWCNTs were first examined by SEM and TEM microscopies. From the SEM analysis, nanotubes were found to grow homogeneously and cover all the substrate surface without showing any precise orientation (data not shown). The best spatial resolution was obtained from high resolution TEM, which made it possible to localize and identify the Cu-nanoparticles on the nanotube surface as shown in Fig. 1. The composition of the nano-partcles was established by performing an electron energy loss spectroscopy (EELS) analysis on several single particles (data not shown). The obtained spectra indicated that nano-particles were made of copper. 


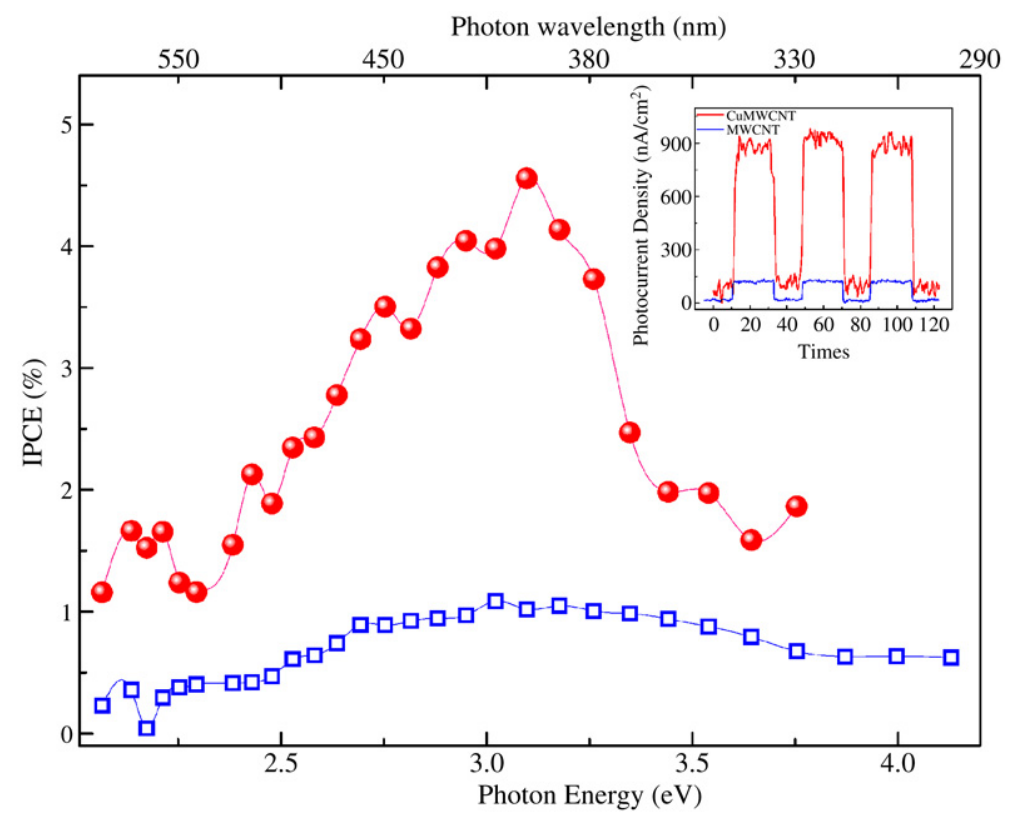

Fig. 2. Photocurrent action spectra of MWCNTs and Cu-MWCNTs obtained as a function of incident photon energy $\left(E_{p h} \lambda>\right.$ $2 \mathrm{eV}$ ). Empty squares $(\square)$ MWCNTs, filled circles ( $\bullet$ Cu-MWCNTs. Electrolyte: NaI $0.5 \mathrm{M}$ and $\mathrm{I}_{2} 0.01 \mathrm{M}$ in acetonitrile. Inset: typical on-off current cycle in MWCNTs and Cu-MWCNTs obtained at $\lambda=400 \mathrm{~nm}$ photon light wavelength.

The Cu nanoparticles were found to be randomly distributed on the external walls of the nanotube and have most often a faceted shape with an (average diameter of about $3.0 \pm 1.0 \mathrm{~nm}$ ). The mean diameter of the grown MWCNTs, as obtained from a statistical analysis performed on several collected images, was $(12 \pm 2) \mathrm{nm}$ and the mean number of walls was $11 \pm 5$.

The MWCNTs electrode performance was obtained by recording the photocurrent density at different incident photon energies. In the process, one of the excited charged species (electrons or holes) is collected at the electrode surface while the other is scavenged by the oxidized or reduced form of the electrolyte in solution. The anodic character of the photocurrent signal as shown in the inset of Fig. 2, indicates the electronic character of the charge carriers.

The photocurrent action spectra (IPCE) [20] obtained from MWCNTs and Cu-MWCNTs normalized to the excited area covered by nanotubes, is reported in Fig. 2 . The photocurrent signal obtained on the bare $\mathrm{SiO}_{2}$ substrate turned out to be structureless and of negligible intensity throughout the photon energy range investigated and was not inserted in the figure. The maximum in the IPCE for bare MWCNTs of $1 \%$ was found at $3.3 \mathrm{eV}$ while it increased up to $4.5 \%$ for $\mathrm{Cu}$ MWCNTs. The maximum of the photocurrent signal around $3.3 \mathrm{eV}$ for MWCNTs is comparable with that reported in earlier experiments performed on similar MWCNT samples [17]. MWCNTs interact similarly to SWCNTs with light showing a peculiar photovoltaic property because they operate as unconventional "Schottky barrier diodes" in which two diode actions occur, one inside the nanotube (between the metallic and semiconductor walls) and the other between the nanotube/nanotube junctions. The enhancement in the overall photocurrent signal for $\mathrm{Cu}-\mathrm{MWCNTs}$ demonstrates the ability of $\mathrm{Cu}$ nanoparticles to significantly modify the electronic properties of the entire hybrid system. Cu nanoparticles are good electron donor upon photo-excitation and improve the interfacial charge-transfer processes with the electrolyte. Therefore, the photocurrent signal increase indicates that MWCNTs promote electrons transfer and transport from the $\mathrm{Cu}$ metal nanoparticles to the numerous concentric cylindrical walls of carbon nanotubes which offer additional pathways for the flow of photo-generated charge carriers.

Working with photoelectrochemical cells can have some drawbacks. It has been observed that the electrolyte can degrade while the solvent quickly evaporates during the spectrum acquisition 
a

Photon Wavelength (nm)

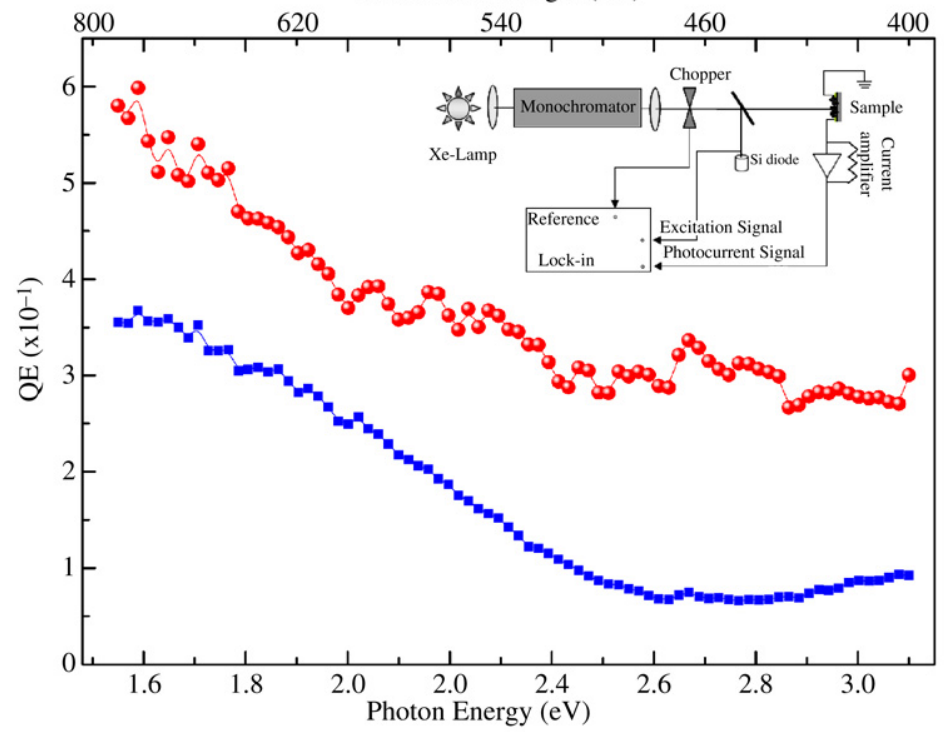

$\mathrm{b}$

Photon Wavelength (nm)

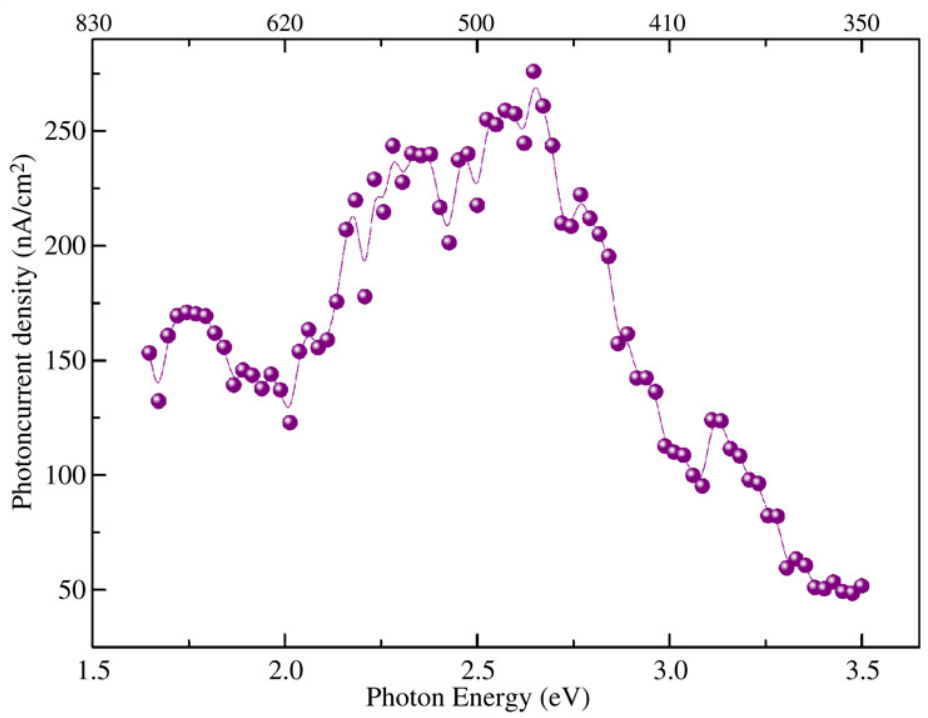

Fig. 3. (a) Calculated quantum efficiency (QE) for MWCNTs and Cu-MWCNTs obtained as a function of incident photon energy $\left(E_{p h}>1.5 \mathrm{eV}\right)$. Filled squares $(\boldsymbol{\square})$ MWCNTs, filled circles $(\bullet) \mathrm{Cu}$ - MWCNTs. Inset: Scheme of the acquisition apparatus. (b) Photocurrent density $(\bullet)$ evaluated by subtracting the MWCNTs current signal from that of Cu-MWCNTs to evidence the $\mathrm{Cu}$-nanoparticles contribution.

time. Although this event never had a significant influence on the collected spectra we reduced the experimental acquisition time to minimize the effect.

In addition, the nanostructures desorption was observed in our samples, probably induced by ion bombardment from the electrolyte. SEM and HRTEM measurements performed on the samples after the photocurrent measurements evidenced that while the CNTs were still well stuck to the sample substrate the $\mathrm{Cu}$ nanoparticles were found to be reduced in quantity. These problems may prevent the long-term stability required for the application of solar cells. 
Photocurrent measurements were also carried out on the same samples with an apparatus working in air without the electrolyte. The signal was collected on each sample, in proximity to the non grounded metallic electrode, as a function of the incident photon energy. The quantum efficiency [21] obtained from the MWCNTs and Cu-MWCNTs normalized by the irradiated sample area, is reported in Fig. 3(a). The signal obtained from the bare silicon dioxide substrate turned out to be structureless and was not reported in the figure. The results obtained show the presence of a photocurrent signal both from MWCNTs and Cu-MWCNTs. The maximum in the QE for bare MWCNTs of $0.35 \%$ was found at $1.6 \mathrm{eV}$ while it increased up to $0.65 \%$ for Cu MWCNTs. The curve reported in Fig. 3(b) was obtained by mathematically subtracting the MWCNTs photocurrent signal from the Cu-MWCNTs one. The line shape obtained in this case reaches a maximum value of $280 \mathrm{nA}$ energy. This results suggests that this measurement is strongly influenced by the $\mathrm{Cu}$-nanoparticles electronic behavior in which the surface plasmons [22] excitation significantly modifies the electronic properties of the entire hybrid system. We note also that decoration of MWCNTs with $\mathrm{Cu}$ nanodots influences all the photocurrent spectrum which extends towards higher energies with respect to the bare MWCNTs. This might be of interest for optoelectronic application of such Cu-MWCNTs based devices.

Although MWCNTs should mimic the electronic properties of metallic graphite, the quantum confinement of the charged carriers within the nanotubes creates strongly-bound excitons which are believed to be responsible for the photocurrent. A detailed explanation of the observed photoresponse as a function of the photon energy based on the optical properties of the CNTs will be published elsewhere [26].

In conclusion the combination of MWCNTs with electron-donor groups constituted by small metal nanoparticles is an innovative concept in the context of photovoltaic systems. MWCNTs are somewhat easier to process than SWCNTs, because they are less prone to forming tight bundles. Due to the presence of numerous concentric cylindrical tubes, MWCNTs should be even more suitable for achieving charge transfer and transport than SWCNTs are. In addition, the ability of Cu nanodots to optically absorb both in the infrared and in the visible range makes this system more suitable for light-energy conversion applications than CNTs functionalized with organic molecules due to higher resistance to continuous optical excitation.

Whereas other systems may have shown higher efficiency, the synthesis method presented can be used for the deposition of other metallic nanoparticles and can be considered complementary to those that already exist. This would include studies on more efficient methods for packing MWCNTs into thin films once each nanotube has been decorated with metallic nanoparticles.

\section{References}

[1] Carbon nanotubes, Acc. Chem. Res. 35 (2002) 997. (special issue).

[2] M.A. Greene, Third Generation Photovoltaics - Advanced Solar Energy Conversion, Springer, Berlin, Germany, 2004.

[3] P.V. Kamat, J. Phys. Chem. C 111 (2007) 2834.

[4] A.C. Khazraji, S. Hotchandani, S. Das, P.V. Kamat, J. Phys. Chem. B 103 (1999) 4693.

[5] S. Barazzouk, S. Hotchandani, K. Vinodgopal, P.V. Kamat, J. Phys. Chem. B 108 (2004) 17015.

[6] D.M. Guldi, G.M.A. Rahman, M. Prato, N.J. Jux, S. Qin, W. Ford, Angew. Chem., Int. Ed. Engl. 44 (2005) 2015.

[7] T. Hasobe, S. Fukuzumi, P. Kamat, JACS 127 (2005) 11884; J. Phys. Chem. B 110 (2006) 25477.

[8] P.V. Kamat, Nanotoday 1 (2006) 20.

[9] A. Kongkanand, R. Martinez Dominguez, P.V. Kamat, Nanoletters 7 (2007) 676.

[10] I. Robel, B. Bunker, P.V. Kamat, Adv. Mater. 17 (2005) 2458.

[11] F. Vietmeyer, B. Seger, P.V. Kamat, Adv. Mater. 19 (2007) 2935.

[12] B. Xue, P. Chen, Q. Hong, J. Lin, K.L. Tan, J. Mater. Chem. 11 (2001) 2378

[13] G.M. Aminur Rahman, D.M. Guldi, E. Zambon, L. Pasquato, N. Tagmatarchis, M. Prato, Small 1 (2005) 527.

[14] C. Bittencourt, A. Felten, J. Ghijsen, J.J. Pireaux, W. Drube, R. Enri, G. Van Tendeloo, Chem. Phys. Lett. 436 (2007) 368; C. Bittencourt, A. Felten, B. Douhard, J.-F. Colomer, G. Van Tendeloo, W. Drube, J. Ghijsen, J.J. Pireaux, Surf. Sci. 601 (2007) 2800.

[15] Y. Cho, C. Kim, H. Moon, Y. Choi, S. Park, C.K. Lee, S. Han, Nanoletters 8 (2007) 81.

[16] A.N. Androitis, M. Menon, G.E. Froudakis, Appl. Phys. Lett. 76 (2000) 3890.

[17] P. Castrucci, F. Tombolini, M. Scarselli, E. Speiser, S. Del Gobbo, W. Richter, M. De Crescenzi, M. Diociaiuti, E. Gatto, M. Venanzi, Appl. Phys. Lett. 89 (2006) 253107.

[18] H.J. Kuhn, S.E. Braslavsky, R. Schmidt, Pure Appl. Chem. 61 (1989) 187.

[19] Because the two metallic Ag electrodes are not symmetric, there is a current flow induced by the built-in-voltage forming a Schottky barrier at the interface between MWCNTs and the metallic electrode. A similar effect has been also observed in case of current flowing through a single wall carbon nanotube suspended between two electrodes upon illumination (see ref. [23-25]). 
[20] The IPCE (photon-to-current efficiency) values was determined from the following equation:

$$
\operatorname{IPCE}(\%)=\frac{100 \cdot i\left(A / \mathrm{cm}^{2}\right) \cdot 1240}{I\left(W / \mathrm{cm}^{2}\right) \cdot \lambda(\mathrm{nm})}
$$

where $i$ is the short circuit photocurrent $\left(\mathrm{A} / \mathrm{cm}^{2}\right), I$ is the incident light intensity $\left(\mathrm{W} / \mathrm{cm}^{2}\right)$ and $\lambda$ is the incident wavelength $(\mathrm{nm})$. See for details reference [4].

[21] The QE (Quantum Efficiency) is defined as $Q E=N_{e-h} / N_{p h}=I_{\text {sample }} \cdot h c /\left(P_{\text {lamp }} \cdot \lambda \cdot e\right)$. Where $N_{e-h}$ is the number of e-h pairs created by incident photons $N_{p h}$ and $P_{\text {lamp }}$ is the power of the incident light at wavelength $\lambda$.

[22] M. De Crescenzi, M. Diociaiuti, L. Lozzi, P. Picozzi, S. Santucci, Solid State Commun. 74 (1990) 115;

S. Di Nardo, L. Lozzi, M. Passacantando, P. Picozzi, S. Cantucci, M. De Crescenzi, Surf. Sci. 307-309 (1994) 922.

[23] K. Kong, S. Han, J. Ihm, Phys. Rev. B 60 (1999) 6074.

[24] M.S. Fuhrer, J. Nygård, L. Shih, M. Forero, Y.-G. Yoon, M.S.C. Mazzoni, H.J. Choi, J. Ihm, S.G. Louie, A. Zettl, P.L. McEuen, Science 288 (2000) 494.

[25] J.U.A. Lee, Appl. Phys. Lett. 87 (2005) 073101.

[26] C. Scilletta, E. Speiser, P. Castrucci, F. Tombolini, M. Scarselli, M. De Crescenzi, M. Simeoni, A. Continenza, B. Delley, 2009, (in preparation). 